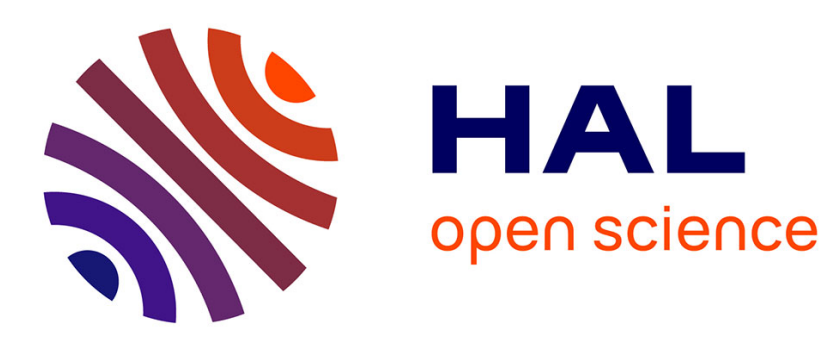

\title{
Pour une éthique des sciences du management: Formation à la connaissance de soi
}

\author{
Florence Rodhain, Angélique Rodhain
}

\section{To cite this version:}

Florence Rodhain, Angélique Rodhain. Pour une éthique des sciences du management: Formation à la connaissance de soi. La Revue des Sciences de Gestion, 2012, 10.3917/rsg.253.0043 hal-01950450

\author{
HAL Id: hal-01950450 \\ https://hal.science/hal-01950450
}

Submitted on 26 Dec 2018

HAL is a multi-disciplinary open access archive for the deposit and dissemination of scientific research documents, whether they are published or not. The documents may come from teaching and research institutions in France or abroad, or from public or private research centers.
L'archive ouverte pluridisciplinaire HAL, est destinée au dépôt et à la diffusion de documents scientifiques de niveau recherche, publiés ou non, émanant des établissements d'enseignement et de recherche français ou étrangers, des laboratoires publics ou privés. 


\title{
Pour une éthique des sciences du management : Formation à la connaissance de soi
}

\author{
par Florence Rodhain et Angélique Rodhain
}

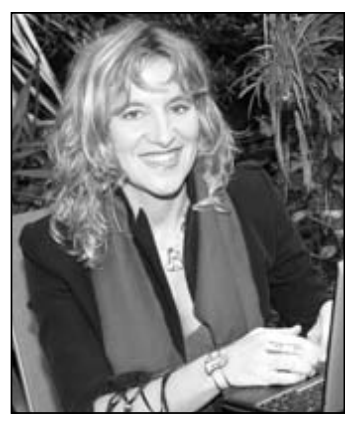

\section{Florence RODHAIN}

Maître de Conférences HDR

MRM - Montpellier Research in Manasement Ecole Polytechnique Universitaire de Montpellier

Université Montpellier 2, France

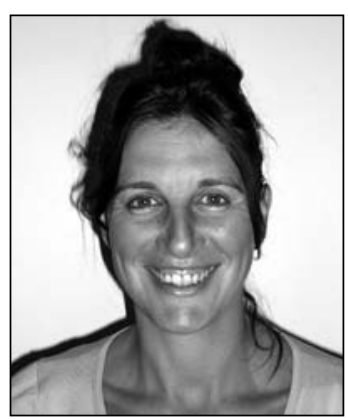

\section{Angélique RODHAIN}

Maître de Conférences

MRM - Montpellier Research in Manasement IUT de Béziers

Université Montpellier 2, France es scandales managériaux semblent se succéder ces dernières années à un rythme effréné : les affaires Enron et WorldCom aux États-Unis, Parmalat en Italie, VivendiUniversal, Rhodia et Société Générale en France, Adecco en Suisse, Khalifa en Algérie... A ces scandales s'ajoute une crise de confiance prenant racine avec l'affaire des subprimes aux États-Unis.

Une question se pose alors : qui a formé les managers ou gestionnaires impliqués dans ces crises et scandales? Qui, sinon les MBA, les grandes écoles, les écoles d'ingénieurs et de commerce? La plupart des cadres mêlés à ces affaires sont diplômés en gestion, et souvent par les plus prestigieuses Business Schools. Dans les années 1990, Enron a embauché chaque année 250 personnes sortant d'un MBA (H. Mintzberg 2004). Dans ces écoles, l'accent est mis sur l'apprentissage des techniques de gestion, laissant de côté la question du sens des actions à entreprendre. Pourtant, sachant que le chiffre d'affaires des multinationales les plus puissantes dépasse largement les budgets de nombreux Etats, la capacité d'action dans la société des managers formés par les MBA et grandes écoles est ainsi considérable.

Les crises récentes ont créé un mouvement de réflexion dans les écoles de commerce et de gestion. Les quelques chercheurs en gestion qui se sont penchés sur ces questions semblent $s$ 'accorder sur le fait que la formation à la gestion ne permet pas de favoriser un comportement éthique chez les futurs cadres (M. Baetz et D. Sharp 2004, R. Kashyap et al. 2006, V. Krishnan 2008, A.M. Lämsä et al. 2007). Par exemple, Venkat Krishnan (2008) s'est intéressé, dans une étude longitudinale de sept ans, à l'impact de l'éducation à la gestion sur les valeurs d'étudiants effectuant un MBA à temps plein sur deux ans. II a pu montrer que cette éducation a changé le système de valeurs des étudiants : les valeurs orientées vers l'individu (par exemple la recherche du confort, du pouvoir, de la reconnaissance, du plaisir) sont devenues plus importantes après le passage par le MBA 
alors que les valeurs orientées vers la collectivité (honnêteté, politesse, harmonie, amitié, paix intérieure et dans le monde) perdaient largement de leur importance après cette éducation. Venkat Krishnan explique ainsi comment l'éducation à la gestion semble rendre les étudiants plus égoïstes, moins concernés par les autres et la société.

Pourtant, dès 1976 (R. Conaway 2000), l'association AACSB (Association to Advance Collegiate Schools of Business) a recommandé aux écoles d'insérer des cours d'éthique dans leurs programmes. Plus récemment, I'AACSB a durci son protocole d'accréditation des écoles se recommandant de l'association, en insistant sur l'importance des cours d'éthique (D. Swanson et W. Frederick 2003). Les scandales récents, particulièrement aux États-Unis, laissent planer un sérieux doute sur la qualité et l'efficacité de ces cours et sur la façon dont ils ont été intégrés dans les programmes (D. Desplaces et al. 2007). II semblerait que le mouvement en faveur de l'enseignement de l'éthique n'ait pas vraiment eu l'ampleur qu'il aurait dû avoir.

Par ailleurs, la prise de conscience récente de la responsabilité des entreprises dans la crise écologique planétaire a fait émerger les concepts de Développement Durable (DD) et de Responsabilité Sociale de l'Entreprise (RSE). Si ces concepts sont enseignés dans certaines écoles, ils le sont souvent dans des diplômes spécialisés, et non pas dans le tronc commun de la formation en gestion. Très largement critiqué par de nombreux chercheurs (O. De Rivero 2003, S. Latouche 2004, E. Gonzales-Gaudiano 2005, A.-C. Martinet 2005, F. Rodhain 2007, L. Sauvé 2007), le Développement Durable arrive, à 24 ans $^{1}$, à l'âge adulte. Fils du "Développement", proche de la retraite (62 ans) ${ }^{2}$, il est venu prêter main forte à l'entreprise familiale : "Le développement durable n'est ni une utopie, ni même une contestation, mais la condition de survie de l'économie de marché " affirmait Louis Schweitzer, Président du CA de Renault ${ }^{3}$ en 2004. Les faits semblent lui donner raison. A "La fin du développement" de François Partant (1999), on a, finement, rétorqué : "développement durable ". Comment, dès lors, interroger la notion même de développement? Certains s'y emploient pourtant, à l'instar de Serge Latouche (2004), inlassable économiste critique, rejoint par des penseurs comme Albert Jacquart (2000) ou Edgar Morin (2009) ("le développement durable? C'est une pensée crétinisée qui met de la vaseline au mot de développement dont elle ne reconnaît pas les carences ${ }^{4}$ ).

Si le Développement Durable (DD) et la Responsabilité Sociale de l'Entreprise (RSE) apportent un véritable engouement dans la société civile, l'ombre de l'épée de Damoclès de leur entière réappropriation par les pouvoirs économico-politiques recouvre ces concepts d'une auréole de grisaille.

Le développement durable peut-il finir par n'être que "le dernier gadget d'une mode managériale qui semble avoir de plus en

1. Le Développement Durable est né officiellement en 1987.

2. La notion de Développement telle qu'entendue aujourd'hui a été utilisée après guerre par Harry S. Truman, président des États-Unis en 1949.

3. Enjeux les Échos, décembre 2004.

4. Edgar Morin, Actes de la conférence débat du réseau intelligence de la complexité, Paris, 25 novembre 2009. plus de difficultés à renouveler ses collections après la décennie financière abrasive qui a clôturé le vingtième siècle" (A.-C. Martinet 2005) ${ }^{5}$ ?

La concrétisation du DD, dans les faits, est critiquable, dans le sens où elle est récupérée par les Pouvoirs en place pour poursuivre en toute impunité des pratiques non durables. Il est difficile d'étudier la notion de DD sans s'intéresser à celle de RSE, elle aussi largement critiquée (C. Aid 2004, J. Allouche et al. 2004, M. Morsing 2006, G. Saint-Lambert 2006, L. Sauvé 2007) car étant considérée, à l'instar du DD, comme trop frileuse, trop consensuelle, faisant office d'un nouveau gadget managérial à la mode. Gilles Saint-Lambert (2006) (un pseudonyme derrière lequel se cache un cadre supérieur en activité dans l'une des directions centrales d'un groupe international dont le siège est situé en France) s'interroge pour savoir si RSE et DD ne seraient pas des stratégies désespérées des grandes entreprises, des idéologies de dernier recours, une façon de "sauver les meubles", des "concepts mous pour temps durs". II s'interroge en conclusion en ces termes : "Ce 'thème de gestion' (la RSE) est-il susceptible de relégitimer l'entreprise ou n'est-il, définitivement, qu'une technique de manipulation destinée à faire accepter le 'froid calcul égoïste' des multinationales? La question est loin d'être résolue...".

II nous paraît, dès lors, peu probable que DD et RSE puissent fondamentalement contribuer à influencer la conscience éthique des étudiants en gestion malgré l'amalgame qui est souvent réalisé entre ces trois termes.

Ainsi, ni l'enseignement de l'éthique, ni celui du DD ou de la RSE ne permettraient de préparer les étudiants aux urgences des crises financières, écologiques et sociales qui se préparent. L'enseignement de l'éthique nous apparaît comme fondamental mais malheureusement certainement pas assez valorisé et reconnu par les autorités scolaires et universitaires.

Dans ce contexte, nous interrogeons l'intérêt d'une pédagogie plus offensive centrée sur la "connaissance de soi ", sans doute originale en pratique mais pourtant pas très novatrice en théorie si l'on considère le célèbre adage de Socrate (470-399 av. J.-C.) : "Connais-toi toi-même".

Dans son article passionnant sur l'impact de l'enseignement de la gestion sur les valeurs des étudiants, Venkat Krishnan écrit (2008) : "The change in value systems of MBA students is not in the direction of taking them towards greater identification with other human beings" (en français : "Le changement dans le système de valeurs des étudiants en MBA ne va pas dans le sens d'une plus grande identification avec les autres êtres humains"). C'est précisément le chemin inverse que nous souhaitons amorcer et étudier : repartir du centre, c'est-à-dire le "soi ", pour ouvrir l'étudiant à l'identification aux autres, ainsi qu'à l'environnement. C'est un chemin vers le décloisonnement, le franchissement des frontières, et le respect de soi, des autres, et de l'environnement.

5. A.C. Martinet (2005), "Le développement sera-t-il durable?", $2^{\text {es }}$ Journées de l'atelier développement durable de l'AIMS, 11 mai. 
Comment enseigner la "connaissance de soi " ? Mais tout d'abord, pourquoi enseigner la "connaissance de soi "?

Cet article conceptuel a pour objectif de plaider pour la formation à la connaissance de soi comme prérequis incontournable à tout cours d'éthique. Pour ce faire, nous proposons dans un premier temps de rappeler les défis à relever qui vont s'imposer aux futurs managers en formation dans les écoles de gestion : défis d'ordre financier, mais également écologique et social, afin, dans un second temps, de montrer l'importance d'un enseignement à la "connaissance de soi" pour faire face à ces défis. Dans un troisième temps, nous interrogerons l'intérêt de ce type de formation pour proposer, dans un quatrième temps, les grandes lignes de cet enseignement à la "connaissance de soi".

\section{Défis d'ordre financier, écologique et social posés aux futurs gestionnaires}

L'absence de moralisation dans les pratiques financières a conduit à des scandales de grande envergure, jusqu'à la crise importante des subprimes aux États-Unis. Ainsi, des défis importants en termes de moralisation (ou re-moralisation) des pratiques bancaires reposent aujourd'hui sur les épaules des étudiants formés à la gestion. Si ces défis sont d'envergure, ils ne sont rien pour James Leape (2008) comparés aux risques écologiques encourus, non seulement par tout un chacun mais aussi et surtout par les entreprises. Les températures n'ont jamais atteint durant le millénaire un tel niveau d'augmentation. Si les scientifiques ont pendant longtemps débattu pour reconnaître ou non l'effet de l'activité humaine sur le changement climatique, cet impact est désormais reconnu scientifiquement. L'organisation IPCC (Intergovernmental Panel on Climate Change) des Nations Unies, qui a reçu le prix Nobel de la Paix pour ses activités, l'explicite en 2007 dans le document "IPCC Summary for PolicyMakers". Avec le rapport de Nicholas Stern (2006), ancien vice-président de la Banque mondiale, ce ne sont plus seulement les scientifiques qui tirent la sonnette d'alarme mais également les économistes et les financiers. Une majorité de scientifiques et d'économistes a ainsi commencé à prendre conscience de ce qui était resté trop longtemps dénoncé par les seuls "écologistes".

Les spécialistes de l'écologie semblent à présent formels : les déséquilibres écologiques sont sans précédent dans l'histoire de I'humanité (Nations Unies 2003, M. Lee 2002, Global Environment Facility 2002, Institut Worldwatch 2006, WWF 2008). Ces perturbations risquent de créer des situations sociales de crise, alors même que nombreux sont les individus à travers la planète souffrant considérablement d'un déséquilibre dans la répartition des richesses. Les populations pauvres en particulier, fragilisées par leur manque de capacité de soulèvement de fonds, risquent en premier d'être touchées violemment par les crises écologiques qui s'annoncent. II s'agira alors d'être en mesure de travailler dans l'urgence et en coopération avec les nations. Les estimations du WWF (2008) énoncent qu'en 2030, si aucune mesure n'est prise pour réguler les modes de vie actuels, les populations terrestres auront besoin de deux planètes pour subvenir à leurs besoins. En 2030, dans I'hypothèse où aucune mesure draconienne ne serait prise pour réguler les dysfonctionnements à venir, c'est $70 \%$ de la planète qui sera détruite et l'on assistera à l'effondrement de l'organisation sociale dans de nombreux pays du globe (conclusion du rapport des Nations Unies préparé par 1100 scientifiques reconnus internationalement - voir Nations Unies, 2003).

Fortes de ces constatations, nous estimons indispensable de réviser certaines des pratiques "éducatives" mises en place depuis plusieurs décennies dans les écoles ou universités qui préparent les cadres de demain, plus particulièrement dans les formations de type MBA.

\section{Lacunes dans l'éducation pour affronter les défis à relever : vers une formation à la connaissance de soi}

Les étudiants sont-ils formés pour affronter les défis écologiques et la récession économique annoncée? Si l'on prend acte des prévisions des spécialistes, les managers devraient avoir à faire face à toujours plus de stress et d'angoisse, à des situations d'urgence, de crise, de remise en cause profonde. Le risque de l'effondrement de l'organisation sociale et écologique annoncé par les scientifiques ayant participé au rapport des Nations Unies (2003) n'apporte-t-il pas l'opportunité (sans par ailleurs tomber dans l'heuristique de la peur) de mener une réflexion profonde sur nos modes de fonctionnement et une remise en cause des modèles de développement, de financiarisation et plus globalement de consommation, qui se sont imposés au niveau planétaire et qui sont directement ou indirectement responsables des dégradations sociales et environnementales? N'est-il pas temps d'interroger le paradigme moderniste qui aurait transformé l'homo economicus en un dangereux perturbateur des équilibres écologiques et sociaux? Les dysfonctionnements qu'il a entraînés ne pourraient-ils pas constituer une opportunité, une invitation à interroger les modes de développement et de croissance responsables en partie des crises actuelles et à venir? Peut-il y avoir croissance sans conscience? La croissance est-elle contre la conscience? Pour qu'il y ait un développement harmonieux, comment le développement de la conscience pourrait-il être favorisé, faisant en sorte de ne pas privilégier uniquement le seul développement économique?

Certains auteurs ont remis en cause l'étroitesse de l'enseignement que reçoit un étudiant en gestion. Ces chercheurs ont particulièrement mis l'accent sur les hypothèses économiques bien trop simplifiantes et étroites sur lesquelles reposent ensuite 
les techniques de gestion enseignées (C. MacLellan et J. Dobson 1997, K. McPhail 2001, J. Pfeffer 2005, R. Kashyap et al. 2006). Sumantra Ghoshal (2005) dénonce, quant à lui, le fait que les écoles de gestion enseignent des théories valorisant l'idée selon laquelle les entreprises doivent rentrer en compétition non seulement avec leurs concurrents, mais également avec leurs propres employés, leurs clients, leurs fournisseurs. En inculquant de telles valeurs idéologiques, les écoles de gestion dépossèdent activement les étudiants, selon Anna Lämsä et al. (2007), de leur capacité à agir dans la société avec responsabilité. Or, si l'on accepte les prévisions des écologistes, il semblerait que les crises à venir exigeront précisément une capacité à réagir, une créativité, une ouverture sur les autres et sur l'environnement, des esprits capables de joindre plutôt que de disjoindre, d'assembler plutôt que de séparer, d'appréhender la complexité plutôt, qu'au mieux, la réduire et au pire, la fuir en la niant. Ce sont ces capacités qui pourraient être inculquées à travers l'éducation à la "connaissance de soi ", en considérant l'éducation, à l'instar de Lucie Sauvé (2004), comme un lieu de développement critique et certainement pas d'endoctrinement (B. Jickling 1999). Selon Paul Laforge (1997), avant d'être un manager éthique, il faut être une personne éthique au quotidien, et cela ne peut passer que par la connaissance de soi.

Pourquoi si peu d'actions sont entreprises, alors qu'un nombre croissant d'individus sont informés de l'état de la planète? Une première piste est l'hypothèse d'un savoir "mutilé ": nous saurions, mais nous ne sentirions pas (F. Rodhain 2008). Un des spécialistes mondiaux en neurologie, Antonio Damasio, explique dans un ouvrage intitulé "l'Erreur de Descartes" (A. Damasio 2001), que les dernières découvertes neurologiques poussent à penser qu'être rationnel, c'est ne pas se couper de ses émotions. Il démontre dans cet ouvrage qu'émotion et raison sont indissociables. On ne peut être rationnel sans être émotionnel. Un savoir intellectuel, coupé du ressenti, serait un savoir mutilé. Un savoir non mutilé serait une connaissance globale, alliant l'émotionnel à l'intellect. L'être humain occidental serait "coupé " : il saurait, mais il ne sentirait plus. Ainsi, l'approche que nous privilégions ici pour prendre en considération les problèmes écologiques serait d'agir au niveau de l'individu, en l'aidant à développer sa conscience, en l'aidant éventuellement à ré-apprendre, à sentir. Car s'il se coupe de sa propre souffrance, alors il ne serait plus apte à sentir la Terre en souffrance et pourrait alors continuer à la mutiler en toute impunité. Les stratégies d'évitement de la souffrance pourraient être la cause de cette coupure. L'individu se couperait de la sensation par un processus de fuite face à la réalité, de façon à éviter l'intolérable. La coupure a pu devenir un geste d'adaptation pour subsister dans un environnement hostile. Une seconde explication du manque de mobilisation devant l'urgence de la crise écologique, économique et sociale serait la spécialisation (F. Rodhain 2008). Si nous sommes coupés au niveau du corps, entre notre ressenti et notre intellect, nous sommes également découpés au niveau des corps de métier. La spécialisation, le découpage à l'extrême favorisent certes l'efficience dans nos modes d'organisation, mais également le réductionnisme, les approches mutilantes et le manque de conscience.

Finalement, si malgré l'urgence annoncée on constate un manque de réaction des systèmes concernés, deux pistes d'explication pourraient être mobilisées : 1) L'individu serait coupé - 2) L'individu serait découpé. La culture occidentale favoriserait, comme le montre Jean-Louis Le Moigne (1990a), la spécialisation dont le risque est le réductionnisme, le manque de vision élargie, et des décisions mutilantes car ne prenant pas en compte la globalité de la situation.

II s'agirait donc, dans les enseignements que nous proposons, premièrement, de chercher une façon de retrouver la sensation pour ré-apprendre à sentir et ne plus seulement comprendre intellectuellement et, deuxièmement, de retrouver l'unité, pour ne plus séparer et ne plus se sentir séparé des autres et de l'environnement.

Ainsi, nous plaidons pour que, de même que l'analyste ne peut soigner autrui qu'après avoir suivi un long travail introspectif, le manager accède à la connaissance de ses propres fonctionnements afin de comprendre et de sentir son environnement, proche comme lointain.

\section{Formation à la connaissance de soi}

Les neurologues, comme les chercheurs de l'Ecole de Palo Alto, nous montrent que l'objectivité est un mythe (J.-P. Changeux 1983, A. Steri 1994, G. Tiry 1994, P. Watzlawick 1976, 1988). Sans rentrer ici dans les détails du processus neurologique d'accès à la réalité, il est établi que toutes les sensations reçues par l'individu ne sont pas analysées car ne recevant pas l'attention du système perceptivo-cognitif (impact des représentations - définies ici comme ce qui est "stocké" dans le cerveau : connaissances, mémoire, histoire personnelle, culture - sur la perception). Pourquoi ne pas supposer également que certaines sensations sont exclues du champ de l'interprétation "sur ordre " des représentations, c'est-à-dire du fait des croyances de l'individu? L'hypothèse avancée est la suivante (F. Rodhain 2008) : les connaissances seraient capables de "couper le canal" de certains types de sensation, du fait des croyances de l'individu, le poussant ainsi à rejeter ce type d'information. La prédominance de la seule raison computée par l'intellect conduit généralement à recaler au second plan des modes de sensation subtils, tel le ressenti corporel. Globalement, le mode de connaissance privilégié est celui où le corps n'est pas considéré comme apportant des informations. C'est l'hypothèse formulée précédemment : la connaissance purement intellectuelle, coupée du corps, serait une connaissance mutilée, duale. Il est une chose de ne pas interpréter les sensations reçues, il en est une autre de couper le canal : est-ce qu'à force de ne plus prendre en compte les sensations subtiles, autres que langagières, dans l'analyse d'une situation, on parviendrait à complètement les ignorer au point de se couper de la source? Si tel était le cas, n'assisterions-nous 
pas alors à un gâchis formidable? Les stimuli seraient multiples, mais du fait de nos croyances, nous nous interdirions de considérer tout un ensemble de stimuli qui nous permettrait peut-être d'approcher la réalité de façon moins mutilante.

Dans le processus d'accès à la réalité, les sensations sont premières : elles alimentent le cerveau. Vient ensuite la perception, qui a pour fonction d'attribuer une signification à la sensation. Ces perceptions peuvent ensuite être transformées en représentations. Cependant, pendant l'acte d'interprétation de la sensation, les représentations agissent. On observe un feed-back : les représentations, connaissances, mémoire, vécu, histoire, interviennent pendant l'interprétation de l'objet observé. Ainsi, nos connaissances et représentations stockées dans notre cerveau brouillent les pistes en produisant un bruit constant. Ces filtres interprétatifs éloignent l'individu de la réalité qu'il cherche à observer. Si la Connaissance existe, l'homme peut-il y avoir accès en utilisant uniquement son mental ? L'observation pure ne serait-elle pas celle d'un esprit dépourvu de représentation? Un tel procédé est-il accessible à l'être humain? Ou bien est-il condamné, par nature, à rester à jamais éloigné de la réalité? La conséquence n'est-elle pas, dès lors, de se connaître en tant que sujet connaissant l'objet? La quête de l'individu étant la connaissance de l'objet, celui-ci peut-il, durant ce processus, faire l'économie de maîtriser la connaissance du sujet qui connaît l'objet? L'objet peut-il être appréhendé si le sujet est dans l'ignorance de lui-même? Apprendre à se connaître en tant que "sujet cherchant à connaître l'objet" ne deviendrait-il pas, dès lors, fondamental ? Cette quête de soi-même se situerait à deux niveaux (F. Rodhain 2008) :

a-apprendre à connaître le fonctionnement de son mental, afin d'éviter de tomber dans ses pièges.

Le mental est un outil merveilleux, précieux, extraordinairement complexe, qui a ouvert des voies fabuleuses au développement de l'être humain. Cependant, ce mental peut nous tendre des pièges qu'il s'agit de débusquer. Pour ce faire, il faut comprendre son fonctionnement : il s'agit alors de s'observer dans l'acte mental. Comment pense-t-on? Quand pense-t-on? Peut-on cesser l'acte de penser? Comment le mental nous empêche-t-il d'être présent à une situation? Comment peut-on s'échapper dans son mental pour fuir la réalité et donc passer à côté de l'objet? Est-il possible de couper le feed-back entre représentations (ou connaissances) et interprétation de l'objet? Si oui, comment? Dans l'objectif de réduire l'influence des représentations dans l'appréhension de l'objet, il s'agit tout d'abord d'être dans la conscience de ses connaissances. Non seulement dans la conscience de ses connaissances, filtres interprétatifs à toute approche de la réalité, mais également dans la conscience de ses processus d'accès à la connaissance. Il y aurait donc un aspect statique (les connaissances stockées) et un aspect dynamique (les processus) face à toute compréhension d'une situation. L'analogie avec l'informatique aboutirait à considérer les connaissances comme les fichiers stockés sur le disque dur de notre cerveau, et les processus comme les programmes informatiques, qui dicteraient à notre insu les logiques interpré- tatives. Ces processus résulteraient d'une inscription, suite à une situation où un certain type de comportement a eu un effet bénéfique important, aboutissant à une généralisation du type "si-alors" comme dans les programmes des systèmes experts. Cette généralisation abusive conduirait à reproduire le processus dans des situations où le contexte est différent de l'environnement originel. L'individu deviendrait alors prisonnier de son programme. Il se penserait libre alors qu'il ne serait que l'instrument de ses processus psychologiques. Pour atteindre la liberté, il devrait alors se défaire des chaînes de ses processus; pour cela il devrait les observer, les disséquer, se libérer de son auto-conditionnement, de son auto-emprisonnement. Ainsi, apprendre à maîtriser son mental signifierait concrètement : être dans la conscience de ses fichiers de connaissances (en évolution constante), et être dans la conscience de ses processus. Cette prise de conscience peut être un préalable à la modification éventuelle, en conscience, de ces fichiers et processus.

$b$ - ré-apprendre à considérer d'autres formes d'appréhension de l'objet.

L'individu serait donc enferré dans ses processus et ses connaissances. II serait condamné à approcher l'objet de façon réduite à l'approche mentale, les autres stimuli disponibles étant ignorés. L'idée serait de cesser de se couper volontairement des stimuli disponibles, dans le but de multiplier les angles d'approche de l'objet, ce qui devrait logiquement conduire à une connaissance plus globale, plus complexe, moins simplifiante.

Ainsi, l'éducation à la connaissance de soi serait également extrêmement bénéfique à la formation du futur manager cherchant à appréhender l'objet de façon la moins mutilante possible. Plus ce dernier aurait conscience de lui, moins il serait mutilant à l'égard de l'objet qu'il observe, car il ne serait pas dupe des processus neurologiques inhérents à sa nature humaine le conduisant à "réduire" l'objet de son observation. II s'agit donc d'ouvrir son esprit à la façon dont il accède à la réalité.

\section{Comment former à la connaissance de soi à I'Université?}

Dans un texte intitulé "De la Réforme de l'Université ", le penseur Edgar Morin (1997) souligne la nécessaire réforme de l'Université, qui doit être non pas programmatique, mais paradigmatique en ce qu'elle concerne notre aptitude à organiser nos connaissances. Il poursuit à ce propos: "Toute réforme de ce type suscite un paradoxe : on ne peut réformer l'institution (les structures universitaires) que si l'on a réformé au préalable les esprits; mais on ne peut réformer les esprits que si l'on a au préalable réformé l'institution. C'est là une impossibilité logique, mais c'est de ce type d'impossibilité logique dont se moque la vie. Qui éduquera les éducateurs? II faut qu'ils s'auto-éduquent et s'éduquent à l'écoute des besoins vivants du siècle, dont sont porteurs aussi les étudiants". 
Gregory Bateson (1980) distingue trois niveaux d'apprentissage. Dans le premier niveau, l'apprentissage apparaît comme une conséquence directe de l'absorption d'informations transmises, qui présentent un intérêt certain, mais qui n'ont aucun impact à long terme sur la vision du monde et sur la personnalité de l'apprenant. Dans le second niveau, l'apprentissage consiste à se ré-approprier les informations transmises de telle façon que le comportement de l'apprenant puisse évoluer. L'apprenant peut ainsi changer une partie de sa vision du monde, mais cela reste cantonné à une partie seulement. Dans le troisième niveau enfin, l'apprentissage apparaît lorsque l'étudiant prend conscience de sa vision du monde en général, de la façon dont ses représentations influencent sa personnalité, de la manière dont il pourrait changer ses représentations ainsi que l'impact que pourraient avoir ces changements sur son identité et sur son développement personnel. Notre approche a l'ambition de se fonder sur le troisième niveau d'apprentissage ainsi défini par Gregory Bateson. A l'instar de Galilée qui affirmait "On ne peut rien apprendre à un homme; mais il est possible de l'aider à découvrir la réponse à l'intérieur de lui ", la démarche du formateur consiste dans cette démarche à aider l'apprenant à se découvrir par lui-même. Les outils que nous préconisons sont de deux natures différentes : des outils théoriques et des outils pratiques. Les outils théoriques sont principalement centrés sur la transmission des ressources de la modélisation systémique, à la fois contextualisante, projective et téléologique (J.-L. Le Moigne 1990a, 1990b) de la pensée complexe (E. Morin 1977, 1980, 1986, 1991, 2001, 2004), et de la connaissance de la connaissance, c'est-à-dire sur les processus neurologiques en œuvre dans l'accès à la réalité. II s'agit de transmettre les dernières connaissances sur l'acquisition du savoir humain, sur le phénomène de construction des connaissances, afin que l'apprenant réalise la façon dont les représentations qu'il se forge influencent sa vision du monde. Ce faisant, il prend alors conscience de sa "subjectivité" dans l'approche de la réalité. Quant à l'adoption de la pensée complexe, celle-ci lui permettra d'élargir le champ de vision, mais aussi de débusquer toutes les pensées simplifiantes et d'adopter comme une seconde nature une attitude consistant à systématiquement chercher une relation causale à toute relation présentée comme linéaire. Subjectivité et complexité sont étroitement liées pour deux raisons principales. Premièrement, moins on a conscience de sa subjectivité, plus le risque est grand d'adopter une approche mutilante, niant la complexité. La neutralité dans l'observation n'existe pas tant que l'apprenant utilise son seul intellect pour approcher la réalité. Moins cette conscience est présente chez l'étudiant, plus il pourra avoir recours avec confiance à des techniques et méthodes réductionnistes. Deuxièmement, l'intellect, seul, n'est qu'un fragment. Si la connaissance intellectuelle est mutilante, comme nous en avons fait l'hypothèse, c'est parce qu'elle est fragmentaire. L'apprenant qui tenterait d'approcher la réalité, avec sa subjectivité se réduisant à son seul intellect, risquerait alors difficilement d'atteindre cette réalité puisque l'intellect ne peut conduire au tout (A. Damasio 2001).
Concernant la partie pratique, elle consiste en une série de mises en situation (individuelle et collective) devant permettre à l'étudiant de prendre conscience :

1) de ses émotions et du rôle qu'elles jouent dans la perception de la réalité et dans les prises de décision quotidiennes;

2) de son corps et du rôle qu'il joue dans la perception de la réalité et dans les prises de décision quotidiennes.

3) de ses représentations (connaissances stockées - aspect statique), de la façon dont elles influencent sa perception de la réalité et de la manière dont elles influencent ses prises de décision;

4) de ses processus psychologiques (aspect dynamique) en œuvre dans l'appréhension de l'objet et de la façon dont ces processus influencent ses prises de décision;

5) de son mode communicationnel, de la façon dont l'apprenant "s'écoute" et "écoute " les autres en situation de communication et de la façon dont il s'exprime, ainsi que de la façon dont ce mode communicationnel influence sa perception de la réalité et ses prises de décision. II s'agit donc de s'exercer en permanence à la critique épistémique interne de toutes ces activités.

E. Jodin (2001) propose de lier une philosophie existentialiste (en considérant les sentiments et croyances des individus) à la philosophie pragmatique par le recours aux cas concrets par le biais de débats en groupe. Pour ce faire, P. Laforge (1997) opte pour un cours d'éthique débutant par la méditation. Cette technique permet, selon l'auteur, d' "arrêter le monde" pour être à l'écoute de son corps et de ses émotions. Le recours à l'art graphique permet ensuite aux apprenants d'accéder à leur système de valeurs et à leurs représentations. Ces représentations apparaissent par la confrontation avec celles des autres. Enfin, des études de cas sont réalisées en utilisant des référents différents tels que la Bible ou des codes de déontologie. L'objectif final étant que chacun ait conscience de posséder une vision servant de guide dans un monde en pleine turbulence. M. Schure, J. Christopher et S. Christopher (2008) ayant utilisé en cours d'éthique les méthodes de connaissance de soi telles que le yoga, la méditation et le qi gong concluent notamment que les apprenants sont plus conscients de leur environnement et plus ouverts à des alternatives de vie et de façons de penser différentes et adoptent de meilleures qualités relationnelles. Quant à D. Koehn (2005), il recourt à la connaissance de soi par une réflexion sur la signification de l'argent car c'est, selon lui, un moyen d'accéder à son système de valeurs. Ces approches, bien que différentes dans la forme, optent toutes pour une plus grande liberté de l'individu qui retrouve son esprit critique et accède à une véritable créativité (E. Jodin, 2001)

\section{Conclusion}

Si les enseignants et chercheurs en économie et sciences de gestion sont amenés à reconnaître l'importance de la responsabilité des managers dans les crises récentes et à identifier comme essentiel que les cadres et dirigeants exercent leur pouvoir 
en toute responsabilité et de façon éthique (E. Freeman 1984, J. Näsi 1995, A. Carroll et A. Buchholtz 2000, B. Joyner et D. Payne 2002, R. Daft 2005, P. Pruzan et M.K. Pruzan 2007), la question de la formation pour parvenir à ces fins reste, quant à elle, une problématique peu étudiée. C'est pourquoi nous désirons activement contribuer à apporter des éléments de réponse à cette problématique de recherche. Or, nous proposons que l'enseignement de l'éthique, au lieu d'être centré sur des aspects théoriques du développement durable ou de la RSE, passe avant tout par une connaissance approfondie de soi afin que l'individu (l'étudiant futur manager, le manager actuel, le chercheur) prenne conscience de son propre fonctionnement intellectuel et émotionnel. Cette connaissance de soi lui permettrait de ne plus être coupé de son environnement (social et écologique) et ainsi de prendre conscience de l'impact de ses propres pensées et actions sur celui-ci. Avant d'être responsable dans ses fonctions managériales, l'individu se doit d'être responsable dans ses actes au quotidien. L'éthique du management ne peut alors faire l'impasse sur les questions d'éthique personnelle. Les cours à la connaissance de soi sont encore utilisés de façon sporadique et il n'existe malheureusement pas encore d'études approfondies démontrant le bien fondé de cet enseignement à long terme sur les apprenants. Parions pourtant que les crises économiques, sociales et environnementales actuelles aboutissent à une généralisation de leur développement.

\section{Bibliographie}

Aid Christian. Behind the Mask: The Real Face of Corporate Social Responsibility. London, 2004, 65 p.

Allouche José, Huault Isabelle et Schmidt Géraldine. Responsabilité sociale des entreprises : la mesure détournée? Congrès AGRH. Montréal, septembre 2004, p. 2390-2410.

Baetz Mark, Sharp David. Integrating Ethics Content into the Core of Business Curriculum: Do Core Teaching Materials Do the Job. Journal of Business Ethics, 2004, n 51, p. 53-63.

Bateson Gregory. Vers une écologie de l'esprit, Tome 1 et 2. Editions du Seuil. Paris, 1980, 299 p. et 346 p.

Carroll Archie, Buchholtz Anne. Business and Society: Ethics and Stakeholder Management (4 ${ }^{\mathrm{e}}$ edition). South-Western College Publishing. Division of Thomson Learning, 2000, 768p.

Changeux Jean-Pierre. L'homme neuronal. Fayard. Paris, 1983, $419 \mathrm{p}$.

Conaway Roger. Ethical Preferences among Business Leaders: Implications for Business Schools. Business Communication Quarterly, 2000, n63, vol 1, p. 23-48.

Daft Richard. Leadership. South-Werstern College Publishing, 2005, 250 p.

Damasio Antonio. L'Erreur de Descartes. Odile Jacob. Paris, 2001, 396 p.
De Rivero Oswaldo. Le Mythe du Développement. Editions Enjeux Planète. Montréal, 2003, 241 p.

Desplaces David, Melchar David, Beauvais Laura et Bosco Susan. The Impact of Business Education on Moral Judgment Competence: An Empirical Study. Journal of Business Ethics, 2007, $\mathrm{n}^{\circ} 74$, p. 73-87.

Freeman Edward. Strategic Management. A Stakeholder Approach. Pitman. HarperCollins. Boston, 1984, 275 p.

Ghoshal Sumantra. Bad Management Theories Are Destroying Good Management Practises. Academy of Management Learning \& Education, 2005, $\mathrm{n}^{\circ}$ 4, vol 1, p. 75-91.

Global Environment Facility. The Challenge of Sustainability. An action agenda for the global environment, Washington D.C., 2002.

Gonzales-Gaudiano Edgar. Education for Sustainable Development: configuration and meaning. Policy Futures in Education, numéro spécial : "Environmental Education and Education for Sustainable Development", 2005, vol.3, n 3, p. 243-250.

http://webarchive.nationalarchives.gov.uk/+/http://www. hm-treasury.gov.uk/stern_review_report.htm

Institut Worldwatch. L'état de la planète. Genève, 2006.

IPCC. IPCC Summary for PolicyMakers. 2007.

Jacquard Albert. J'accuse l'économie triomphante. Le livre de poche, 2000, $188 \mathrm{p}$.

Jickling Bob. Beyond Sustainability: Should We Expect More From Education? Southern African Journal of Environmental Education, 1999, n¹9, p. 60-67.

Jodin Emmanuelle. L'enseignement de l'éthique dans les écoles de gestion : la nécessaire integration. Cahier de Recherche OIPG, 2001, n²001-01, 20p.

Joyner Branda, Payne Dinah. Evolution and Implementation: A Study of Values Business Ethics and Corporate Social Responsibility. Journal of Business Ethics, 2002, n 41, p. 297-312.

Kashyap Rajiv, Mir Raza et lyer Easwar. Toward Responsive Pedagogy: Linking Social Responsibility to Firm Performance Issues in the Classroom. Academy of Management Learning \& Education, 2006, n 5, vol 3, p. 366-376.

Koehn Daryl. Transforming our students : teaching business ethics post-enron. Business Ethics Quarterly, 2005, $\mathrm{n}^{\circ} 15$, vol. 1, p. $137-151$.

Krishnan Venkat. Impact of MBA Education On Student's Values: Two Longitudinal Studies. Journal of Business Ethics, 2008, $\mathrm{n}^{\circ} 83$, p. 233-246.

Laforge Paul. Teaching business ethics through meditation. Journal of Business Ethics, 1997, n 16, vol. 12-13, p. 1283-1295.

Lämsä Anna Maija, Vehkaperä Meri, Puttonen Tuomas et Pesonen Hanna-Leena. Effect of Business Education on Women and Men Student's Attitudes on Corporate Responsibility in Society. Journal of Business Ethics, 2007, n 82, pp.45-58. 
Latouche Serge. Survivre au développement : De la décolonisation de l'imaginaire économique à la construction d'une société alternative. Editions Mille et une nuits. Paris, 2004, 126p.

Le Moigne Jean-Louis. La modélisation des systèmes complexes. Afcet systèmes. Editions Dunod. Paris, 1990b, 178 p.

Le Moigne Jean-Louis. La théorie du système général. Théorie de la modélisation. P.U.F., $3^{e}$ édition (première édition : 1977). 1990a, 258 p.

Leape James, Directeur général du WWF International, Rapport Planète vivante 2008, $46 \mathrm{p}$.

Lee Matilda. L'Etat de la planète 2002. L'Ecologiste, 2002, n 8, octobre, p. 25-30.

MacLellan Cheryl et Dobson John. Women, Ethics, and MBAs. Journal of Business Ethics, 1997, n 16, p. 1201-1209.

Martinet Alain-Charles. Le développement sera-t-il durable ? $2^{\text {es }}$ journées de l'atelier développement durable de I'AIMS, 11 mai 2005.

McPhail Ken. The Other Objective of Ethics Education: Re-Humanising the Accounting Profession - A Study of Ethics Education in Law, Engineering, Medicine and Accountancy. Journal of Business Ethics, 2001, n 34, p. 279-298.

Mintzberg Henry. Managers Not MBAs: A Hard Look at the Soft Practice of Managing and Management Development. Financial Times. Prentice Hall. New York, 2004, 480 p.

Morin Edgar, De la réforme de l'Université, la double mission, Congrès de Locarno, 30 avril - 2 mai 1997 : Annexes au document de synthèse CIRET-UNESCO disponible sur :

http://a.e.t.free.fr/article.php?id_article = 16 consulté le 19/02/12.

Morin Edgar. Ethique (La Méthode : tome 6). Seuil. Paris, 2004, 271 p.

Morin Edgar. Introduction à la pensée complexe. E.S.F., Collection communication et complexité. Paris, 1990, 158 p.

Morin Edgar. L'identité humaine (La Méhode : tome 5). Seuil. Paris, 2001, 384 p.

Morin Edgar. La Connaissance de la Connaissance (La Méthode : tome 3). Seuil. Paris, 1986, 236 p.

Morin Edgar. La Nature de la Nature (La Méthode : tome 1). Seuil. Paris, 1977, 399 p.

Morin Edgar. La Vie de la Vie (La Méthode : tome 2). Seuil. Paris, 1980, $470 \mathrm{p}$.

Morin Edgar. Les Idées. Leur habitat, leur vie, leurs moeurs, leur organisation (La Méthode : tome 4). Seuil. Paris, 1991, 261 p.

Morin Edgar. Quelle Université pour demain ? Congrès International CIRET. Locarno, Suisse, 30 avril-2 mai 1997.

Morsing Mette. Corporate Social Responsibility as Strategic Auto-Communication: On The Role of External Stakeholders for Member Identification. Business Ethics, 2006, n 15, p. 171-182.
Näsi Juha (ed.). Understanding Stakeholder Thinking. LSR-Publications, Helsinki, 1995.

Nations Unies. The Global Environment Outlook, 2003.

Partant François, La fin du développement naissance d'une alternative?, Actes du Sud, 1999, Collection Babel, 252 p.

Pfeffer Jeffrey. Why Do Bad Management Theories Persit? A Comment on Ghoshal. Academy of Management Learning \& Education, 2005, n 4, vol 1, p. 96-100.

Pruzan Peter et Mikkelsen Kirsten Pruzan. Leading with Wisdom - Spiritual-based Leadership in Business. Sage publications. 2007, 352 p.

Rodhain Florence. Changer les mots à défaut de soigner les maux? Critique du développement durable. Revue Française de Gestion (RFG), 2007, n 176, vol 33, p. 203-210.

Rodhain Florence. Le 'Je' et le décryptage du jeu du mental comme préalable à une science en conscience. HDR Soutenue le 9 avril 2008, Université Montpellier 2, 175 p.

Saint-Lambert Gilles. Quand l'entreprise s'affiche "responsable"... Education Permanente, 2006, n 167, p. 1-10.

Sauvé Lucie. Entre culture commune et diversité culturelle. Actes du Colloque International pour l'Éducation à l'Environnement pour un Développement Durable. Muséum d'Histoire Naturelle de Paris, 14 et 15 avril 2004.

Sauvé Lucie. L'équivoque du développement durable. Chemin de Traverse, $2007, n^{\circ} 4$, p. 31-47.

Schure Marc, Christopher John et Christopher Suzanne. Mind-Body medecine and the art of self-care teaching mindfulness to counseling students through yoga, meditation and qi gong. Journal of Counseling \& Development, 2008, $n^{\circ} 86$, p. 47-56.

Stern Nicholas, Stern review final report on the Economics of Climate Change, publié le 30 octobre 2006, disponible sur :

Streri Arlette. Comment l'homme perçoit-il le monde? L'homme cognitif, direction : A.Weil-Barais, P.U.F. Paris, 1994, p. 91-193.

Swanson Diane, et Frederick William. Campaingn AACSB: Are Business Schools Complicit in Corporate Corruption? Journal of Individual Employment Rights, 2003, n 10, p. 151-165.

Tiry Gérard. Connaître le réel. Chronique Sociale. Lyon, 1994, $120 \mathrm{p}$.

Watzlawick Paul. L'invention de la réalité. Contributions au constructivisme. Direction : P.Watzlawick, Seuil. 1988, 373 p.

Watzlawick Paul. La réalité de la réalité. Seuil. 1976, 237 p.

WWF. Rapport Planète vivante 2008, 46 p. 
Copyright of Revue des Sciences de Gestion is the property of Direction et Gestion Sarl and its content may not be copied or emailed to multiple sites or posted to a listserv without the copyright holder's express written permission. However, users may print, download, or email articles for individual use. 